\title{
Effect of Micro and Bulk Solvation on the Mechanism of Nucleophilic Substitution at Sulfur in Disulfides
}

\author{
Joseph M. Hayes and Steven M. Bachrach* \\ Department of Chemistry \\ Trinity University \\ 1 Trinity Place \\ San Antonio, TX 78212
}

\section{Supporting Information Available}

Table S1 and coordinates of all optimized B3LYP/6-31+G* structures, their absolute energies, and number of imaginary frequencies. This material is available free of charge via the Internet at http://pubs.acs.org.

Table S1. B3LYP/6-31+G* Relative Energies $\left(\mathrm{kcal} \mathrm{mol}^{-1}\right)$ for the cis and trans (in parentheses) Pathways of Reactions 1-3 with Different Levels of Water Solvation.

\begin{tabular}{|c|c|c|c|}
\hline Solvation Level & Reactants & $\underline{\text { TS }}$ & $\underline{\text { INT }}$ \\
\hline \multicolumn{3}{|c|}{ Reaction 1 } \\
\hline $1 \mathrm{H} 2 \mathrm{O}$ & 0.0 & $-10.9(-10.9)$ & $-14.1(-14.2)$ \\
\hline $2 \mathrm{H} 2 \mathrm{O}$ & 0.0 & $-7.5(-7.9)$ & $-10.3(-10.6)$ \\
\hline $3 \mathrm{H} 2 \mathrm{O}$ & 0.0 & $-5.7(-6.1)$ & $-7.8(-7.6)$ \\
\hline \multicolumn{3}{|c|}{ Reaction 2} \\
\hline $1 \mathrm{H} 2 \mathrm{O}$ & 0.0 & $-10.2(-10.2)$ & $-12.7(-12.6)$ \\
\hline $2 \mathrm{H} 2 \mathrm{O}$ & 0.0 & $-7.1(-7.5)$ & $-9.1(-9.5)$ \\
\hline $3 \mathrm{H} 2 \mathrm{O}$ & 0.0 & $-4.5(-5.0)$ & $-6.3(-6.1)$ \\
\hline \multicolumn{4}{|c|}{ Reaction 3 } \\
\hline $1 \mathrm{H} 2 \mathrm{O}$ & 0.0 & $-6.5(-6.5)$ & $-8.6(-8.6)$ \\
\hline $2 \mathrm{H} 2 \mathrm{O}$ & 0.0 & $-4.6(-5.2)$ & $-5.1(-5.7)$ \\
\hline $3 \mathrm{H} 2 \mathrm{O}$ & 0.0 & $-2.8(-3.2)$ & $-3.1(-3.3)$ \\
\hline
\end{tabular}


TS-1 cis

$\begin{array}{lrrr}\mathrm{O} & 0.000000 & 0.000000 & 0.000000 \\ \mathrm{H} & 0.000000 & 0.000000 & 0.987271 \\ \mathrm{H} & 0.958758 & 0.000000 & -0.199432 \\ \mathrm{O} & 0.618393 & 0.358412 & 2.737356 \\ \mathrm{H} & 1.544338 & 0.298836 & 2.420365 \\ \mathrm{H} & 0.476257 & 1.334676 & 2.737122 \\ \mathrm{~S} & 3.033367 & 1.243854 & 0.534161 \\ \mathrm{H} & 4.361586 & 1.019445 & 0.657584 \\ \mathrm{~S} & 2.725244 & 3.395671 & -1.995097 \\ \mathrm{H} & 2.986923 & 2.068125 & -1.933578 \\ \mathrm{~S} & 2.568485 & 3.694008 & -4.111296 \\ \mathrm{H} & 3.861494 & 4.003542 & -4.373685 \\ \mathrm{O} & -0.485110 & 2.738330 & -0.395688 \\ \mathrm{H} & -0.407904 & 1.752660 & -0.341204 \\ \mathrm{H} & 0.257928 & 3.010762 & -0.962941 \\ \mathrm{O} & 0.721586 & 3.124735 & 2.152180 \\ \mathrm{H} & 1.629524 & 2.919646 & 1.844284 \\ \mathrm{H} & 0.199821 & 3.128464 & 1.315810 \\ \mathrm{E}(\mathrm{B} 3 \mathrm{~L} Y \mathrm{P})=-1502.201595 & \mathrm{NIMAG}=1\end{array}$

\section{TS-1 trans}

$\begin{array}{lrrr}\mathrm{O} & 0.000000 & 0.000000 & 0.000000 \\ \mathrm{H} & 0.000000 & 0.000000 & 0.987141 \\ \mathrm{H} & 0.960272 & 0.000000 & -0.196210 \\ \mathrm{O} & 0.646380 & 0.358480 & 2.731915 \\ \mathrm{H} & 1.565975 & 0.304092 & 2.394237 \\ \mathrm{H} & 0.503621 & 1.333812 & 2.745956 \\ \mathrm{~S} & 3.038832 & 1.223380 & 0.507412 \\ \mathrm{H} & 4.355229 & 0.929640 & 0.607793 \\ \mathrm{~S} & 2.706360 & 3.266623 & -2.108811 \\ \mathrm{H} & 2.929414 & 1.934539 & -2.018229 \\ \mathrm{~S} & 2.381726 & 3.489715 & -4.214364 \\ \mathrm{H} & 1.049645 & 3.245940 & -4.253327 \\ \mathrm{O} & -0.400313 & 2.753619 & -0.405355 \\ \mathrm{H} & -0.364214 & 1.766443 & -0.337157 \\ \mathrm{H} & 0.391465 & 2.989087 & -0.922713 \\ \mathrm{O} & 0.763399 & 3.134865 & 2.168382 \\ \mathrm{H} & 1.671962 & 2.925785 & 1.866500 \\ \mathrm{H} & 0.247579 & 3.140366 & 1.328688 \\ \mathrm{E}(\mathrm{B} 3 \mathrm{~L} Y \mathrm{P})=-1502.202300 & \mathrm{NIMAG}=1\end{array}$

\section{INTw-1 cis}

$0 \quad 0.000000$

$\mathrm{H} \quad 0.000000$

$\mathrm{H} \quad 0.956009$

O 0.596492

$\mathrm{H} \quad 1.532451$

$\mathrm{H} \quad 0.486680$

S 3.035964

$\mathrm{H} \quad 4.376696$

S 2.930941

$\mathrm{H} \quad 2.955300$

S 2.830396

$\mathrm{H} \quad 4.160713$

o -0.386143

$\mathrm{H}-0.369448$

$\mathrm{H} \quad 0.403507$

O 0.724924

$\mathrm{H} \quad 1.646212$

H 0.225642

0.000000

0.000000

0.000000

0.000000

0.986538

0.367432

0.263995

1.349911

1.314157

1.141515

3.079046

$1.881158-2.330641$

$4.234429-3.669548$

$4.439995-3.810172$

$2.769442-0.390228$

$1.782480-0.341416$

$2.996956-0.915959$

$3.113484 \quad 2.179600$

$2.957720 \quad 1.887877$

$3.130632 \quad 1.327467$

$\mathrm{E}(\mathrm{B} 3 \mathrm{LYP})=-1502.204050 \mathrm{NIMAG}=0$

\section{INTw-1 trans}

$\begin{array}{lrrr}\mathrm{O} & 0.000000 & 0.000000 & 0.000000 \\ \mathrm{H} & 0.000000 & 0.000000 & 0.986770 \\ \mathrm{H} & 0.956259 & 0.000000 & -0.205656 \\ \mathrm{O} & 0.589943 & 0.361655 & 2.746718 \\ \mathrm{H} & 1.527645 & 0.265662 & 2.491835 \\ \mathrm{H} & 0.473924 & 1.343428 & 2.726109 \\ \mathrm{~S} & 3.026176 & 1.337817 & 0.485659 \\ \mathrm{H} & 4.369558 & 1.188999 & 0.471038 \\ \mathrm{~S} & 2.879819 & 3.111208 & -1.725155 \\ \mathrm{H} & 2.888217 & 1.907249 & -2.322607 \\ \mathrm{~S} & 2.752502 & 4.255917 & -3.669990 \\ \mathrm{H} & 1.403647 & 4.306558 & -3.770292 \\ \mathrm{O} & -0.377176 & 2.767666 & -0.400615 \\ \mathrm{H} & -0.367632 & 1.780722 & -0.345056 \\ \mathrm{H} & 0.443980 & 2.984979 & -0.881998 \\ \mathrm{O} & 0.698021 & 3.111801 & 2.191936 \\ \mathrm{H} & 1.621248 & 2.962813 & 1.901844 \\ \mathrm{H} & 0.201085 & 3.127444 & 1.339033 \\ \mathrm{E}(\mathrm{B} 3 \mathrm{LYP})=-1502.204412 & \mathrm{NIMAG}=0\end{array}$


TSw-1 cis

$\begin{array}{lrrr}\mathrm{O} & 0.000000 & 0.000000 & 0.000000 \\ \mathrm{H} & 0.000000 & 0.000000 & 0.993931 \\ \mathrm{H} & 0.938394 & 0.000000 & -0.242930 \\ \mathrm{~S} & 2.994408 & 2.171297 & 1.927761 \\ \mathrm{~S} & 4.668859 & 0.537144 & 2.077162 \\ \mathrm{~S} & 1.145388 & 4.104415 & 2.021023 \\ \mathrm{O} & 0.069552 & 0.374726 & 2.732176 \\ \mathrm{H} & -0.626280 & 1.069549 & 2.837754 \\ \mathrm{H} & 0.917006 & 0.860772 & 2.755452 \\ \mathrm{O} & -1.827200 & 2.456507 & 2.672303 \\ \mathrm{H} & -1.314387 & 3.233111 & 2.956954 \\ \mathrm{H} & -1.774545 & 2.510717 & 1.683701 \\ \mathrm{H} & 1.847293 & 5.057069 & 1.367206 \\ \mathrm{H} & 5.598824 & 1.218594 & 1.368670 \\ \mathrm{O} & -1.096124 & 2.635362 & -0.006315 \\ \mathrm{H} & -0.335192 & 3.165169 & 0.324419 \\ \mathrm{H} & -0.727228 & 1.735811 & -0.160269 \\ \mathrm{H} & 3.354609 & 2.711627 & 3.103076 \\ \mathrm{E}(\mathrm{B} 3 \mathrm{~L} Y \mathrm{P})=-1502.202619 & \mathrm{NIMAG}=1\end{array}$

TSw-1 trans

$\begin{array}{rrrr}\mathrm{O} & 0.000000 & 0.000000 & 0.000000 \\ \mathrm{H} & 0.000000 & 0.000000 & 0.992507 \\ \mathrm{H} & 0.938049 & 0.000000 & -0.246327 \\ \mathrm{~S} & 2.786159 & 2.236291 & 1.617557 \\ \mathrm{~S} & 4.403465 & 0.540664 & 1.452987 \\ \mathrm{~S} & 1.039477 & 4.216865 & 2.012148 \\ \mathrm{O} & 0.071959 & 0.410362 & 2.739348 \\ \mathrm{H} & -0.668743 & 1.057251 & 2.844726 \\ \mathrm{H} & 0.876613 & 0.964359 & 2.698923 \\ \mathrm{O} & -1.916511 & 2.403660 & 2.674197 \\ \mathrm{H} & -1.428420 & 3.199118 & 2.949856 \\ \mathrm{H} & -1.862254 & 2.450586 & 1.685050 \\ \mathrm{H} & 1.612280 & 5.125178 & 1.190265 \\ \mathrm{H} & 3.810221 & -0.341580 & 2.290907 \\ \mathrm{O} & -1.168254 & 2.607367 & 0.010133 \\ \mathrm{H} & -0.434205 & 3.166788 & 0.352446 \\ \mathrm{H} & -0.761674 & 1.725901 & -0.152280 \\ \mathrm{H} & 3.391037 & 2.784159 & 2.684176 \\ \mathrm{E}(\mathrm{B} 3 \mathrm{~L} Y \mathrm{P})=-1502.202711 & \mathrm{NIMAG}=1\end{array}$

\section{INT-1 cis}

$0 \quad 0.000000$

$\mathrm{H} \quad 0.000000$

$\mathrm{H} \quad 0.952958$

S 2.635608

$\mathrm{H} \quad 3.939116$

S 3.329188

S 2.156543

O 0.155169

$\mathrm{H}-0.327303$

$\mathrm{H} \quad 1.091453$

O -0.949985

$\mathrm{H}-0.164933$

$\mathrm{H}-0.964793$

$\mathrm{H} \quad 2.026206$

$\mathrm{H} \quad 3.255112$

O -0.619719

$\mathrm{H} \quad 0.280839$

$\mathrm{H}-0.482662$

$E(B 3 L Y P)=-1502.2070228$ NIMAG $=0$ 


\section{TS-2 cis}

o $2.352053-1.095613-1.921861$

$\mathrm{H} \quad 3.183268-0.874100-1.438851$

$\mathrm{H} \quad 1.838516-0.265271-1.821081$

$\begin{array}{lllll}0 & 4.269862 & 0.003824 & -0.141417\end{array}$

$\mathrm{H} \quad 3.673123 \quad 0.781945-0.198462$

$\mathrm{H} \quad 3.918077-0.446411 \quad 0.662146$

S $1.213125 \quad 1.403491-0.102650$

$\begin{array}{llll}\mathrm{S}-1.643725 & -0.134706 & 0.406027\end{array}$

$\mathrm{H}-1.158675 \quad 0.431860 \quad-0.723844$

S $-3.543466-0.846944-0.262789$

○ $1.072193-2.548598 \quad 0.115414$

H $\quad 1.499203-2.137541-0.677894$

$\mathrm{H} \quad 0.216751 \quad-2.090437 \quad 0.195520$

o $2.566200-0.928595 \quad 1.924738$

$\mathrm{H} \quad 2.066683-0.108657 \quad 1.719281$

$\mathrm{H} \quad 2.072396-1.618689 \quad 1.423965$

C $\quad 0.749612 \quad 3.183399-0.013487$

$\mathrm{H} \quad 1.038455 \quad 3.710203-0.931403$

$\mathrm{H}-0.334058 \quad 3.299902 \quad 0.119277$

H $1.246721 \quad 3.674322 \quad 0.832072$

$\begin{array}{llll}\text { C }-4.618041 & 0.611319 & 0.032517\end{array}$

$\mathrm{H}-4.570662 \quad 0.922803 \quad 1.079853$

$\mathrm{H}-4.333516 \quad 1.448814-0.611391$

$\mathrm{H}-5.643098 \quad 0.305362-0.207761$ $E(B 3 L Y P)=-1580.823864 \quad \mathrm{NIMAG}=1$

\section{TS-2 trans}

0.000000

$\mathrm{H} \quad 0.000000$

H 0.961487

O 0.639888

H 1.557888

H 0.483774

S 3.016601

S 2.683804

H 2.848907

S 2.423910

O -0.455402

$\mathrm{H}-0.399385$

H 0.347306

○ 0.736040

$\mathrm{H} \quad 1.632040$

H 0.198885

C 4.836856

H 5.075222

H 5.341689

H 5.252860

C 0.627431

H 0.372897

$\mathrm{H} \quad 0.035638$

$\mathrm{H} \quad 0.400551$

$\mathrm{E}(\mathrm{B} 3 \mathrm{LYP})=-1580.824838 \mathrm{NIMAG}=1$

$0.000000 \quad 0.987437$

$0.000000-0.194913$

$0.360086 \quad 2.729687$

$0.320211 \quad 2.380943$

$\begin{array}{ll}1.333490 & 2.740779\end{array}$

1.238676

0.526461

$3.220572-2.074761$

$1.877827-2.055157$

$3.573541-4.166138$

$2.739040-0.431626$

$1.754006-0.348040$

$2.979740-0.929552$

$\begin{array}{ll}3.130780 & 2.137159\end{array}$

$2.895769 \quad 1.809736$

$3.138829 \quad 1.311941$

$\begin{array}{ll}-0.086979 & 0.747204\end{array}$

$1.350998-0.260546$

$\begin{array}{lll}1.510074 & 1.504178\end{array}$

$3.273301-4.397307$

$2.227878-4.202234$

$3.919374-3.743468$

\section{INTw-2 cis}

0.000000

H 0.000000

$\mathrm{H} \quad 0.958190$

$0.000000 \quad 0.000000$

$0.000000 \quad 0.986170$

0.653785

$0.000000-0.203390$

0.366126

2.736770

$\mathrm{H} \quad 1.573928$

H 0.530250

0.283119

2.414387

S 3.045343

$\begin{array}{ll}1.346301 & 2.722828\end{array}$

1.2691130 .455466

S 2.878586

$3.047119-1.772409$

$\mathrm{H} \quad 2.871994$

$1.834561-2.355065$

S 2.835734

O -0.411071

$\mathrm{H}-0.381357$

$4.183115-3.695961$

$2.763744-0.394958$

$1.777227-0.339941$

H 0.370976

$2.994555-0.930172$

O 0.789812

3.114450

2.147199

$\mathrm{H} \quad 1.691045$

2.906837

1.819620

$\mathrm{H} \quad 0.256832$

3.134379

1.316994

C 4.869728

H 5.206862

1.036594

0.450367

H 5.180239

0.634180

1.413221

$\mathrm{H} \quad 5.375068$

$0.340152-0.338237$

C 4.632569

1.994813

0.280871

$\mathrm{H} \quad 5.133095$

$4.373253-4.015265$

\begin{tabular}{l}
$5.104866 \quad 3.409760-4.233802$ \\
\hline .745952
\end{tabular}

$\mathrm{H} \quad 4.745952 \quad 5.029539-4.886310$

$\mathrm{E}(\mathrm{B} 3 \mathrm{LYP})=-1580.825268 \mathrm{NIMAG}=0$

\section{INTw-2 trans}

\subsection{0}

$\mathrm{H} \quad 0.000000$

0.000000

0.000000

0.9784190 .639778

$\mathrm{H} \quad 0.960823$

0.000000

0.985926

$0 \quad 0.682630$

0.000000

$-0.198832$

$\mathrm{H} \quad 1.593994$

$\mathrm{H} \quad 0.597565$

S 3.074595

0.357612

2.735046

0.239858

2.397212

$1.341184 \quad 2.725222$

S 2.960763

1.153761

0.431780

H 2.898536

$2.944235-1.842596$

S 2.909768

O -0.276082

$1.715663-2.388093$

$\mathrm{H}-0.314756$

$4.034985-3.775336$

$\mathrm{H} \quad 0.544271$

$2.779194-0.407672$

O 0.931415

$1.793789-0.333488$

$\mathrm{H} \quad 1.823223$

$2.942372-0$.

3.105541

$-0.910748$

$\mathrm{H} \quad 0.398147$

2.859133

2.145925

C 4.875705

3.139597

1.819299

$\mathrm{H} \quad 5.226821$

0.782383

1.317258

H 5.097249

0.429896

0.372694

$\mathrm{H} \quad 5.440921$

1.349998

$\begin{array}{lll}1.0921 & 1.683443 & 0.107499\end{array}$

C 1.104765

$\mathrm{H} \quad 0.639617$

$\mathrm{H} \quad 0.610805$

$\mathrm{H} \quad 0.974567$

$-0.370155$

$4.193029-4.071493$

$3.216431-4.237761$

$4.686944-3.229597$

$E(B 3 L Y P)=-1580.825796$ NIMAG $=0$ 
TSw-2 cis

$\begin{array}{lrrr}\mathrm{O} & 0.000000 & 0.000000 & 0.000000 \\ \mathrm{H} & 0.000000 & 0.000000 & 0.992473 \\ \mathrm{H} & 0.940115 & 0.000000 & -0.239730 \\ \mathrm{~S} & 2.830986 & 2.254164 & 1.473171 \\ \mathrm{~S} & 4.460380 & 0.639891 & 1.092013 \\ \mathrm{~S} & 1.079125 & 4.193995 & 2.030670 \\ \mathrm{O} & 0.104414 & 0.399387 & 2.738741 \\ \mathrm{H} & -0.605409 & 1.077574 & 2.860155 \\ \mathrm{H} & 0.932176 & 0.914806 & 2.685214 \\ \mathrm{O} & -1.819019 & 2.457571 & 2.725196 \\ \mathrm{H} & -1.276232 & 3.232612 & 2.959862 \\ \mathrm{H} & -1.809544 & 2.487504 & 1.735255 \\ \mathrm{O} & -1.130864 & 2.633716 & 0.031903 \\ \mathrm{H} & -0.389599 & 3.173998 & 0.394237 \\ \mathrm{H} & -0.740839 & 1.745799 & -0.135039 \\ \mathrm{H} & 3.446049 & 2.710041 & 2.578130 \\ \mathrm{C} & 1.826821 & 5.586748 & 1.092869 \\ \mathrm{H} & 2.235018 & 5.231832 & 0.139084 \\ \mathrm{H} & 1.064953 & 6.346663 & 0.881667 \\ \mathrm{H} & 2.636640 & 6.059465 & 1.661703 \\ \mathrm{C} & 5.575033 & 1.591132 & -0.013445 \\ \mathrm{H} & 6.315804 & 0.898578 & -0.430654 \\ \mathrm{H} & 5.012227 & 2.043579 & -0.836727 \\ \mathrm{H} & 6.101288 & 2.382430 & 0.531939 \\ \mathrm{E}(\mathrm{B} 3 \mathrm{~L} \mathrm{IP})=-1580.823185 & \mathrm{NIMAG}=1\end{array}$

TSw-2 trans

$\begin{array}{rrrr}\mathrm{O} & 0.000000 & 0.000000 & 0.000000 \\ \mathrm{H} & 0.000000 & 0.000000 & 0.991700 \\ \mathrm{H} & 0.938928 & 0.000000 & -0.245530 \\ \mathrm{~S} & 2.768171 & 2.146664 & 1.497115 \\ \mathrm{~S} & 4.284856 & 0.426417 & 1.150661 \\ \mathrm{~S} & 1.098501 & 4.180145 & 2.035763 \\ \mathrm{O} & 0.076952 & 0.392244 & 2.750204 \\ \mathrm{H} & -0.655413 & 1.047331 & 2.867724 \\ \mathrm{H} & 0.884497 & 0.940894 & 2.701623 \\ \mathrm{O} & -1.854315 & 2.437396 & 2.727169 \\ \mathrm{H} & -1.316938 & 3.208077 & 2.985874 \\ \mathrm{H} & -1.820457 & 2.481168 & 1.737415 \\ \mathrm{O} & -1.122728 & 2.642591 & 0.057179 \\ \mathrm{H} & -0.378196 & 3.175967 & 0.425884 \\ \mathrm{H} & -0.735977 & 1.756717 & -0.124810 \\ \mathrm{H} & 3.502451 & 2.698593 & 2.479304 \\ \mathrm{C} & 1.864416 & 5.533282 & 1.053393 \\ \mathrm{H} & 2.695442 & 5.997074 & 1.598411 \\ \mathrm{H} & 2.247032 & 5.148774 & 0.100695 \\ \mathrm{H} & 1.118767 & 6.308351 & 0.838859 \\ \mathrm{C} & 3.838001 & -0.721534 & 2.514089 \\ \mathrm{H} & 4.388202 & -1.657657 & 2.361562 \\ \mathrm{H} & 4.114844 & -0.308753 & 3.490465 \\ \mathrm{H} & 2.765766 & -0.940673 & 2.513160 \\ \mathrm{E}(\mathrm{B} 3 \mathrm{~L} \mathrm{P})=-1580.823200 & \mathrm{NIMAG}=1\end{array}$

\section{INT-2 cis}

$\begin{array}{llll}0 & -1.687662 & -2.092028 & -1.072343\end{array}$

$\mathrm{H}-1.746983-2.062866-0.085631$

$\mathrm{H}-0.719627-2.155299-1.248591$

S $\quad 1.088475 \quad-0.019874 \quad 0.360241$

H $\quad 2.350016-0.073855 \quad 0.819345$

S $\quad 1.650905-1.921177-1.166837$

S $\quad 0.678598 \quad 1.910969 \quad 1.844625$

$\begin{array}{llll}0 & -1.730731 & -1.600585 & 1.707363\end{array}$

H $-2.145703-0.702477 \quad 1.656353$

$\begin{array}{llll}\mathrm{H} & -0.779177 & -1.398752 & 1.763888\end{array}$

$\begin{array}{llll}0 & -2.544128 & 1.028107 & 1.241472\end{array}$

$\mathrm{H}-1.669108 \quad 1.440137 \quad 1.422182$

$\begin{array}{llll}\mathrm{H} & -2.612055 & 0.952240 & 0.262644\end{array}$

C $\quad 1.019378 \quad 3.256789 \quad 0.642795$

H $\quad 2.092081 \quad 3.340018 \quad 0.433936$

$\mathrm{H} \quad 0.493708 \quad 3.079415 \quad-0.302252$

$\begin{array}{llll}\mathrm{H} & 0.670327 & 4.207162 & 1.063794\end{array}$

C $\quad 1.877314 \quad-0.943699-2.704557$

$\mathrm{H} \quad 1.074263-0.206095 \quad-2.817638$

$\mathrm{H} \quad 2.836227-0.412922-2.703150$

$\mathrm{H} \quad 1.852612-1.619694-3.567565$

$\begin{array}{llll}0 & -2.704987 & 0.399875 & -1.545532\end{array}$

$\mathrm{H}-2.081236 \quad 0.859302-2.126038$

$\mathrm{H}-2.343071-0.526498-1.456865$

$\mathrm{E}(\mathrm{B} 3 \mathrm{LYP})=-1580.826128 \mathrm{NIMAG}=1$

\begin{tabular}{|c|c|c|c|}
\hline \multicolumn{4}{|c|}{ INT-2 trans } \\
\hline $\mathrm{H}$ & 0.000000 & 0.000000 & 0.000000 \\
\hline S & 0.000000 & 0.000000 & 1.342791 \\
\hline S & 2.487087 & 0.000000 & 1.238375 \\
\hline S & -2.487087 & 0.000000 & 1.238375 \\
\hline C & 2.779650 & 1.809845 & 1.108005 \\
\hline C & -2.779650 & -1.809845 & 1.108005 \\
\hline $\mathrm{H}$ & 2.405379 & 2.203278 & 0.156081 \\
\hline $\mathrm{H}$ & -2.405379 & -2.203278 & 0.156081 \\
\hline $\mathrm{H}$ & 2.292565 & 2.352451 & 1.926079 \\
\hline $\mathrm{H}$ & -2.292565 & -2.352451 & 1.926079 \\
\hline $\mathrm{H}$ & 3.858093 & 2.000297 & 1.162747 \\
\hline $\mathrm{H}$ & -3.858093 & -2.000297 & 62747 \\
\hline 0 & 2.007999 & -0.064467 & 4.575488 \\
\hline O & -2.007999 & 0.064467 & 4.575488 \\
\hline 0 & 0.015441 & 1.945825 & 4.345395 \\
\hline 0 & -0.015441 & -1.945825 & 4.345395 \\
\hline $\mathrm{H}$ & 1.411255 & 0.721305 & 4.588505 \\
\hline $\mathrm{H}$ & -1.411255 & -0.721305 & 4.588505 \\
\hline $\mathrm{H}$ & 2.352477 & -0.091160 & 3.653628 \\
\hline $\mathrm{H}$ & -2.352477 & 0.091160 & 3.653628 \\
\hline $\mathrm{H}$ & -0.764284 & 1.359856 & 4.533420 \\
\hline $\mathrm{H}$ & 0.764284 & -1.359856 & 4.533420 \\
\hline $\mathrm{H}$ & 0.009161 & 2.017080 & 3.375770 \\
\hline $\mathrm{H}$ & & -2.017080 & 3.375770 \\
\hline & $(B 3 L Y P)=-1$ & 580.827373 & VIMAG =0 \\
\hline
\end{tabular}




\section{TS-3 cis}

$\begin{array}{crrr}\mathrm{O} & 0.000000 & 0.000000 & 0.000000 \\ \mathrm{H} & 0.000000 & 0.000000 & 0.986706 \\ \mathrm{H} & 0.963880 & 0.000000 & -0.188826 \\ \mathrm{O} & 0.728865 & 0.373386 & 2.709687 \\ \mathrm{H} & 1.622486 & 0.320160 & 2.302323 \\ \mathrm{H} & 0.589033 & 1.348374 & 2.724795 \\ \mathrm{~S} & 3.062850 & 1.158405 & 0.400229 \\ \mathrm{H} & 4.323539 & 0.715273 & 0.613556 \\ \mathrm{~S} & 2.434124 & 3.545016 & -2.420364 \\ \mathrm{~S} & 1.802081 & 4.698961 & -4.077970 \\ \mathrm{H} & 3.009410 & 5.140597 & -4.512653 \\ \mathrm{O} & -0.468866 & 2.734127 & -0.393766 \\ \mathrm{H} & -0.386895 & 1.748437 & -0.331605 \\ \mathrm{H} & 0.237435 & 3.004228 & -1.006930 \\ \mathrm{O} & 0.875754 & 3.145049 & 2.090864 \\ \mathrm{H} & 1.760886 & 2.886022 & 1.757145 \\ \mathrm{H} & 0.320272 & 3.139729 & 1.277269 \\ \mathrm{C} & 2.758060 & 1.916094 & -3.193226 \\ \mathrm{H} & 1.869069 & 1.552394 & -3.715139 \\ \mathrm{H} & 3.607356 & 1.975146 & -3.880506 \\ \mathrm{H} & 2.996821 & 1.263333 & -2.345102 \\ \mathrm{E}(\mathrm{B} 3 \mathrm{~L} \mathrm{P})=-1541.516345 & \mathrm{NIMAG}=1\end{array}$

\section{TS-3 trans}

$\begin{array}{crrr}\mathrm{O} & 0.000000 & 0.000000 & 0.000000 \\ \mathrm{H} & 0.000000 & 0.000000 & 0.986753 \\ \mathrm{H} & 0.963331 & 0.000000 & -0.189575 \\ \mathrm{O} & 0.718398 & 0.361106 & 2.713703 \\ \mathrm{H} & 1.617061 & 0.313561 & 2.318711 \\ \mathrm{H} & 0.575514 & 1.335701 & 2.737600 \\ \mathrm{~S} & 3.039984 & 1.214766 & 0.404190 \\ \mathrm{H} & 4.328179 & 0.839578 & 0.579976 \\ \mathrm{~S} & 2.467868 & 3.393126 & -2.379665 \\ \mathrm{~S} & 1.754388 & 4.606062 & -3.971682 \\ \mathrm{H} & 0.420849 & 4.420494 & -3.809663 \\ \mathrm{O} & -0.379801 & 2.753751 & -0.418792 \\ \mathrm{H} & -0.350823 & 1.767349 & -0.336232 \\ \mathrm{H} & 0.421877 & 2.975710 & -0.928110 \\ \mathrm{O} & 0.850058 & 3.138699 & 2.136355 \\ \mathrm{H} & 1.741010 & 2.893843 & 1.805931 \\ \mathrm{H} & 0.307324 & 3.146001 & 1.314822 \\ \mathrm{C} & 2.555773 & 1.751720 & -3.192315 \\ \mathrm{H} & 1.563388 & 1.422328 & -3.512965 \\ \mathrm{H} & 3.240151 & 1.782370 & -4.044587 \\ \mathrm{H} & 2.932546 & 1.083980 & -2.411051 \\ \mathrm{E}(\mathrm{B} 3 \mathrm{~L} \mathrm{P})=-1541.51735 & \mathrm{NIMAG}=1\end{array}$

\section{INTw-3 cis}

$0-1.877420-2.377050$

$\mathrm{H}-1.886264-2.384393$

Н $-0.914248-2.379669$

$0-1.187294-2.040839$

$\mathrm{H}-0.277532-2.125273$

$\mathrm{H}-1.300515-1.061011$

S $1.167911-1.194473$

H $\quad 2.480275-1.519516 \quad 1.162885$

S $\quad 0.979204 \quad 0.978472-1.283851$

S $\quad 0.700152 \quad 2.525959-2.768905$

$\mathrm{H} \quad 2.002244 \quad 2.811439-3.017559$

$\begin{array}{llll}0 & -2.204033 & 0.394077 & 0.297111\end{array}$

$\begin{array}{llll}\mathrm{H} & -2.191457 & -0.593812 & 0.346992\end{array}$

$\mathrm{H}-1.412164 \quad 0.622265 \quad-0.224240$

$\begin{array}{llll}0 & -1.013632 & 0.723244 & 2.855815\end{array}$

$\begin{array}{llll}\mathrm{H} & -0.111725 & 0.501827 & 2.542695\end{array}$

$\begin{array}{llll}\mathrm{H} & -1.535650 & 0.743633 & 2.019443\end{array}$

$\begin{array}{llll}\text { C } \quad 1.087016 & -0.477880 & -2.400387\end{array}$

$\mathrm{H} \quad 0.203667-0.534112-3.042056$

$\mathrm{H} \quad 1.992074-0.425580 \quad-3.013413$

$\mathrm{H} \quad 1.131955-1.343317-1.735187$

$\mathrm{E}(\mathrm{B} 3 \mathrm{LYP})=-1541.5165367 \mathrm{NIMAG}=0$

\section{INTw-3 trans}

$\begin{array}{lrrr}\mathrm{O} & 0.000000 & 0.000000 & 0.000000 \\ \mathrm{H} & 0.000000 & 0.000000 & 0.986697 \\ \mathrm{H} & 0.962869 & 0.000000 & -0.190911 \\ \mathrm{O} & 0.717515 & 0.344868 & 2.717461 \\ \mathrm{H} & 1.618021 & 0.277897 & 2.330839 \\ \mathrm{H} & 0.595558 & 1.322411 & 2.743779 \\ \mathrm{~S} & 3.058642 & 1.173879 & 0.418129 \\ \mathrm{H} & 4.347325 & 0.783371 & 0.550300 \\ \mathrm{~S} & 2.631487 & 3.322267 & -2.255799 \\ \mathrm{~S} & 2.059837 & 4.654044 & -3.823043 \\ \mathrm{H} & 0.712038 & 4.542643 & -3.725112 \\ \mathrm{O} & -0.307235 & 2.769529 & -0.405939 \\ \mathrm{H} & -0.312844 & 1.782686 & -0.328001 \\ \mathrm{H} & 0.510154 & 2.965645 & -0.901287 \\ \mathrm{O} & 0.901267 & 3.122616 & 2.160740 \\ \mathrm{H} & 1.792466 & 2.872493 & 1.836047 \\ \mathrm{H} & 0.366233 & 3.143182 & 1.334138 \\ \mathrm{C} & 2.653542 & 1.726686 & -3.162090 \\ \mathrm{H} & 1.654537 & 1.473108 & -3.528196 \\ \mathrm{H} & 3.361615 & 1.770918 & -3.994168 \\ \mathrm{H} & 2.972895 & 0.992427 & -2.417100 \\ \mathrm{E}(\mathrm{B} 3 \mathrm{~L} \mathrm{PP})=-1541.517352 & \mathrm{NIMAG}=0\end{array}$


TSw-3 cis

$\begin{array}{lrrr}\mathrm{O} & 0.000000 & 0.000000 & 0.000000 \\ \mathrm{H} & 0.000000 & 0.000000 & 0.993880 \\ \mathrm{H} & 0.938954 & 0.000000 & -0.241419 \\ \mathrm{~S} & 3.205241 & 1.862329 & 2.109392 \\ \mathrm{~S} & 4.590400 & 0.080831 & 1.888292 \\ \mathrm{~S} & 1.273108 & 3.966999 & 2.164305 \\ \mathrm{O} & 0.086112 & 0.357874 & 2.735433 \\ \mathrm{H} & -0.582102 & 1.079161 & 2.848835 \\ \mathrm{H} & 0.952022 & 0.811034 & 2.756111 \\ \mathrm{O} & -1.723890 & 2.517495 & 2.717554 \\ \mathrm{H} & -1.144835 & 3.255316 & 2.985006 \\ \mathrm{H} & -1.690385 & 2.572980 & 1.729403 \\ \mathrm{H} & 2.007270 & 4.972233 & 1.634056 \\ \mathrm{H} & 5.618536 & 0.734519 & 1.297211 \\ \mathrm{O} & -0.983311 & 2.696613 & 0.030637 \\ \mathrm{H} & -0.203507 & 3.175630 & 0.396491 \\ \mathrm{H} & -0.659820 & 1.782857 & -0.135642 \\ \mathrm{C} & 3.815570 & 2.377349 & 3.772139 \\ \mathrm{H} & 3.148210 & 3.171210 & 4.111305 \\ \mathrm{H} & 3.785579 & 1.528694 & 4.460210 \\ \mathrm{H} & 4.840712 & 2.753513 & 3.699383 \\ \mathrm{E}(\mathrm{B} 3 \mathrm{~L} \mathrm{P})=-1541.513904 & \mathrm{NIMAG}=1\end{array}$

TSw-3 trans

$\begin{array}{crrr}\mathrm{O} & 0.000000 & 0.000000 & 0.000000 \\ \mathrm{H} & 0.000000 & 0.000000 & 0.993625 \\ \mathrm{H} & 0.937900 & 0.000000 & -0.245312 \\ \mathrm{~S} & 3.040158 & 1.984063 & 2.137739 \\ \mathrm{~S} & 4.459873 & 0.249162 & 1.868065 \\ \mathrm{~S} & 1.044104 & 4.061020 & 2.254486 \\ \mathrm{O} & 0.057850 & 0.368014 & 2.736972 \\ \mathrm{H} & -0.672258 & 1.027561 & 2.845989 \\ \mathrm{H} & 0.877084 & 0.903413 & 2.740867 \\ \mathrm{O} & -1.905157 & 2.389239 & 2.705030 \\ \mathrm{H} & -1.386553 & 3.161050 & 2.997981 \\ \mathrm{H} & -1.842336 & 2.460493 & 1.718808 \\ \mathrm{H} & 1.697578 & 5.047483 & 1.598374 \\ \mathrm{H} & 3.758586 & -0.683385 & 2.556273 \\ \mathrm{O} & -1.080882 & 2.660663 & 0.060027 \\ \mathrm{H} & -0.339980 & 3.164919 & 0.472622 \\ \mathrm{H} & -0.708951 & 1.769188 & -0.121992 \\ \mathrm{C} & 3.661452 & 2.498381 & 3.795536 \\ \mathrm{H} & 3.094727 & 3.389620 & 4.069510 \\ \mathrm{H} & 3.492820 & 1.705605 & 4.530389 \\ \mathrm{H} & 4.729941 & 2.722925 & 3.737394 \\ \mathrm{E}(\mathrm{B} 3 \mathrm{~L} \mathrm{P})=-1541.514311 & \mathrm{NIMAG}=1\end{array}$

\section{INT-3 cis}

$\begin{array}{lrrr}\mathrm{O} & 0.000000 & 0.000000 & 0.000000 \\ \mathrm{H} & 0.000000 & 0.000000 & 0.988026 \\ \mathrm{H} & 0.953329 & 0.000000 & -0.232957 \\ \mathrm{~S} & 2.739377 & 2.544075 & 1.314343 \\ \mathrm{~S} & 3.306834 & 0.720948 & -0.300071 \\ \mathrm{~S} & 1.984828 & 4.351699 & 2.916810 \\ \mathrm{O} & 0.194248 & 0.451558 & 2.777051 \\ \mathrm{H} & -0.339373 & 1.286940 & 2.813219 \\ \mathrm{H} & 1.114442 & 0.769961 & 2.765932 \\ \mathrm{O} & -1.055940 & 2.927623 & 2.553840 \\ \mathrm{H} & -0.286529 & 3.484463 & 2.805746 \\ \mathrm{H} & -1.035009 & 2.935761 & 1.567654 \\ \mathrm{H} & 1.845065 & 5.316330 & 1.977791 \\ \mathrm{H} & 3.232085 & 1.493593 & -1.408526 \\ \mathrm{O} & -0.535677 & 2.713733 & -0.228775 \\ \mathrm{H} & 0.378869 & 3.042432 & -0.185652 \\ \mathrm{H} & -0.428396 & 1.726945 & -0.258340 \\ \mathrm{C} & 4.521442 & 3.000782 & 1.494029 \\ \mathrm{H} & 4.630607 & 3.564731 & 2.421261 \\ \mathrm{H} & 5.116187 & 2.086494 & 1.523188 \\ \mathrm{H} & 4.844031 & 3.614516 & 0.647224 \\ \mathrm{E}(\mathrm{B} 3 \mathrm{LYP})=-1541.517264 & \mathrm{NIMAG}=0\end{array}$

\section{INT-3 trans}

$\begin{array}{rrrr}\mathrm{O} & 0.000000 & 0.000000 & 0.000000 \\ \mathrm{H} & 0.000000 & 0.000000 & 0.987690 \\ \mathrm{H} & 0.954151 & 0.000000 & -0.231227 \\ \mathrm{~S} & 2.757949 & 2.496594 & 1.335246 \\ \mathrm{~S} & 3.322923 & 0.616451 & -0.204716 \\ \mathrm{~S} & 2.008024 & 4.368870 & 2.885877 \\ \mathrm{O} & 0.200457 & 0.453726 & 2.782782 \\ \mathrm{H} & -0.335558 & 1.288171 & 2.813711 \\ \mathrm{H} & 1.118828 & 0.777292 & 2.775838 \\ \mathrm{O} & -1.033837 & 2.930932 & 2.545644 \\ \mathrm{H} & -0.260952 & 3.481066 & 2.802491 \\ \mathrm{H} & -1.007826 & 2.938263 & 1.559210 \\ \mathrm{H} & 1.803420 & 5.282223 & 1.907310 \\ \mathrm{H} & 3.670924 & -0.268676 & 0.759096 \\ \mathrm{O} & -0.517778 & 2.717330 & -0.232628 \\ \mathrm{H} & 0.401280 & 3.034527 & -0.200412 \\ \mathrm{H} & -0.421373 & 1.730014 & -0.265089 \\ \mathrm{C} & 4.536506 & 2.994143 & 1.405839 \\ \mathrm{H} & 4.600652 & 3.911734 & 1.992226 \\ \mathrm{H} & 5.125990 & 2.206345 & 1.882791 \\ \mathrm{H} & 4.908193 & 3.155337 & 0.391654 \\ \mathrm{E}(\mathrm{B} 3 \mathrm{y})\end{array}$

$\mathrm{E}(\mathrm{B} 3 \mathrm{LYP})=-1541.516934 \mathrm{NIMAG}=0$ 


$\begin{array}{lrrr}\text { TSi-3 cis } \\ \mathrm{O}-1.661012 & -2.171185 & -1.287999 \\ \mathrm{H} & -1.640516 & -2.179768 & -0.300155 \\ \mathrm{H} & -0.713487 & -2.149372 & -1.545145 \\ \mathrm{~S} & 1.079077 & 0.350111 & -0.012009 \\ \mathrm{~S} & 1.606752 & -1.402614 & -1.739582 \\ \mathrm{~S} & 0.369407 & 2.024761 & 1.743166 \\ \mathrm{O} & -1.459766 & -1.726852 & 1.487840 \\ \mathrm{H} & -2.011379 & -0.903930 & 1.525280 \\ \mathrm{H} & -0.547782 & -1.385618 & 1.467488 \\ \mathrm{O} & -2.719172 & 0.752292 & 1.283885 \\ \mathrm{H} & -1.941324 & 1.271205 & 1.584242 \\ \mathrm{H} & -2.660349 & 0.783279 & 0.299497 \\ \mathrm{H} & 0.180729 & 3.045944 & 0.875068 \\ \mathrm{H} & 1.435973 & -0.590254 & -2.809117 \\ \mathrm{O} & -2.212120 & 0.535656 & -1.507552 \\ \mathrm{H} & -1.295536 & 0.859011 & -1.527620 \\ \mathrm{H} & -2.111613 & -0.452713 & -1.528193 \\ \mathrm{C} & 2.867317 & 0.754787 & 0.237823 \\ \mathrm{H} & 2.950215 & 1.809142 & 0.504264 \\ \mathrm{H} & 3.283590 & 0.143188 & 1.043321 \\ \mathrm{H} & 3.400359 & 0.545761 & -0.690457 \\ \mathrm{E}(\mathrm{B} 3 \mathrm{~L} \mathrm{P})=-1541.516236 & \mathrm{NIMAG}=1\end{array}$

TSi-3 trans

$\begin{array}{lrrr}\mathrm{O} & 0.000000 & 0.000000 & 0.000000 \\ \mathrm{H} & 0.000000 & 0.000000 & 0.986930 \\ \mathrm{H} & 0.954791 & 0.000000 & -0.232792 \\ \mathrm{~S} & 2.862413 & 2.490830 & 1.234164 \\ \mathrm{~S} & 3.318237 & 0.547244 & -0.303296 \\ \mathrm{~S} & 2.234077 & 4.350693 & 2.811445 \\ \mathrm{O} & 0.242147 & 0.455694 & 2.785887 \\ \mathrm{H} & -0.242484 & 1.321371 & 2.817220 \\ \mathrm{H} & 1.177659 & 0.722191 & 2.768713 \\ \mathrm{O} & -0.859362 & 2.992685 & 2.556289 \\ \mathrm{H} & -0.062555 & 3.516545 & 2.792550 \\ \mathrm{H} & -0.857050 & 2.995055 & 1.569097 \\ \mathrm{H} & 1.986791 & 5.275471 & 1.853384 \\ \mathrm{H} & 3.683387 & -0.320288 & 0.669592 \\ \mathrm{O} & -0.440394 & 2.734205 & -0.231513 \\ \mathrm{H} & 0.491718 & 3.013515 & -0.237393 \\ \mathrm{H} & -0.384679 & 1.743548 & -0.260300 \\ \mathrm{C} & 4.635110 & 3.017336 & 1.196785 \\ \mathrm{H} & 4.679904 & 4.097554 & 1.340659 \\ \mathrm{H} & 5.195332 & 2.523062 & 1.996204 \\ \mathrm{H} & 5.053871 & 2.737580 & 0.229125 \\ \mathrm{E}(\mathrm{B} 3 \mathrm{~L} \mathrm{P})=-1541.516600 & \mathrm{NIMAG}=1\end{array}$

